\title{
Bioinspired Fabrication of Calcium-doped TiP Coating with Nanofibrous Microstructure to accelerate osseointegration
}

Bianyun Caial, Peijie Tanª ${ }^{\mathrm{a}}$, Nan Jianga ${ }^{\mathrm{a}}$ Zhijun Guo ${ }^{\mathrm{b}}$, Birol Ayc ${ }^{\mathrm{c}}$, Shujun Lid ${ }^{\mathrm{d}}$ Yi Hou ${ }^{\mathrm{a}}$, Yubao Li Yanjun You ${ }^{\mathrm{e}}$, Li Zhang ${ }^{\mathrm{a}}$, Songsong Zhu ${ }^{\text {a* }}$

a Analytical \& Testing Center; State Key Laboratory of Oral Diseases \& National Clinical Research Center for Oral Disease \& West China Hospital of Stomatology, Sichuan University, Chengdu 610065, China

${ }^{\mathrm{b}}$ School of Materials Science and Physics, China University of Mining and Technology, Xuzhou 221116, China

${ }^{\mathrm{c}}$ Institute of Biomaterials and Biomedical Engineering, University of Toronto, Toronto, Ontario, M5S 3G9, Canada

${ }^{\mathrm{d}}$ Institute of Metal Research, Chinese Academy of Sciences, 72 Wenhua Road, Shenyang 110016, China.

e Sichuan Institute for Food and Drug Control, Chengdu 610065, China

*Corresponding authors

E-mail: zhangli9111@126.com (Li Zhang); zss_1977@163.com (Songsong Zhu)

\section{ASSOCIATED CONTENT}
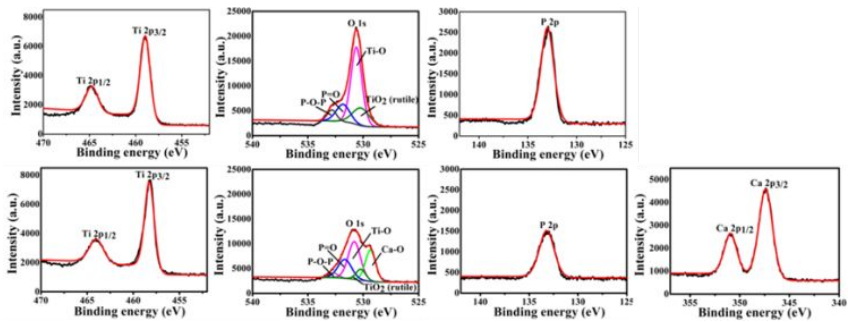

Fig. S1. The XPS high resolution spectra for $\mathrm{Ti}_{2 \mathrm{p}}, \mathrm{O}_{1 \mathrm{~s}}$ and $\mathrm{P}_{2 \mathrm{p}}$ of the TiP coating (up) and for $\mathrm{Ti}_{2 \mathrm{p}}, \mathrm{O}_{1 \mathrm{~s}}, \mathrm{P}_{2 \mathrm{p}}$ and $\mathrm{Ca}_{2 \mathrm{p}}$ of the Ca-TiP coating (below).

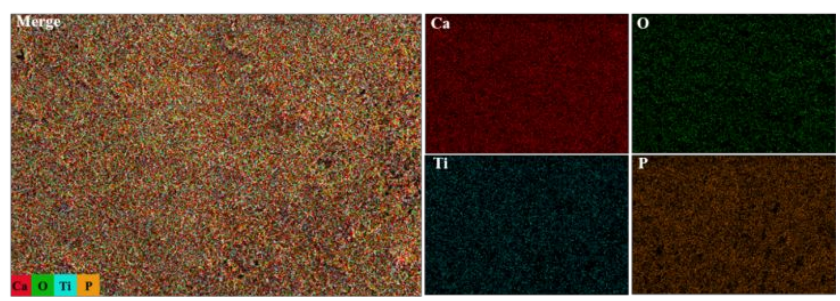

Fig. S2. The elemental mappings of Ti, P, O and Ca for sample of Ca-TiP.

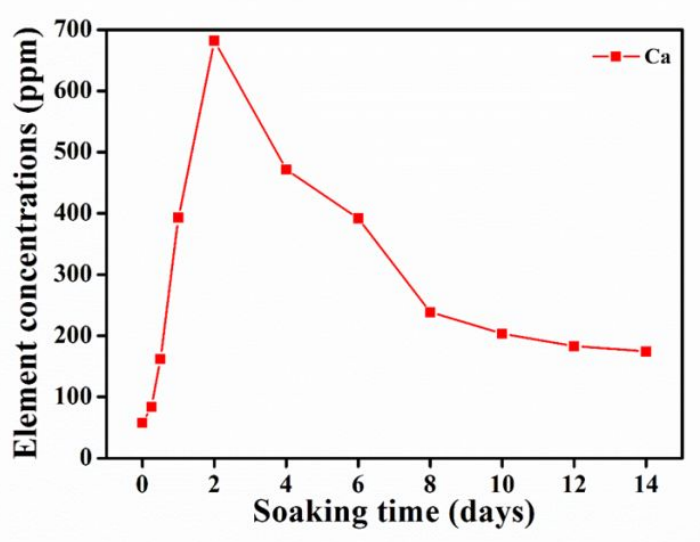

Fig. S3. ICP-OES analysis for the change of $\mathrm{Ca}^{2+}$ concentrations for Ca-TiP soaking in culture medium for $6 \mathrm{~h}, 12 \mathrm{~h}, 1 \mathrm{~d}, 2 \mathrm{~d}, 4 \mathrm{~d}, 6 \mathrm{~d}, 8 \mathrm{~d}, 10 \mathrm{~d}, 12 \mathrm{~d}$, and $14 \mathrm{~d}$.

\section{Ion Dissolution from the Ca-TiP Coatings}

The release curves in Fig. S3 showed that concentrations of $\mathrm{Ca}^{2+}$ rose significantly after two days of soaking, then decreased sharply over the next 6 days until kept declining steady during subsequent periods. In addition, there was still about $18.7 \%$ of $\mathrm{Ca}^{2+}$ left by the end of the test, confirming a continuous delivery for bioactive ions. The sustained release of $\mathrm{Ca}^{2+}$ can provide a saturated ion environment around the implant, helping accelerate the nucleation of apatite. 


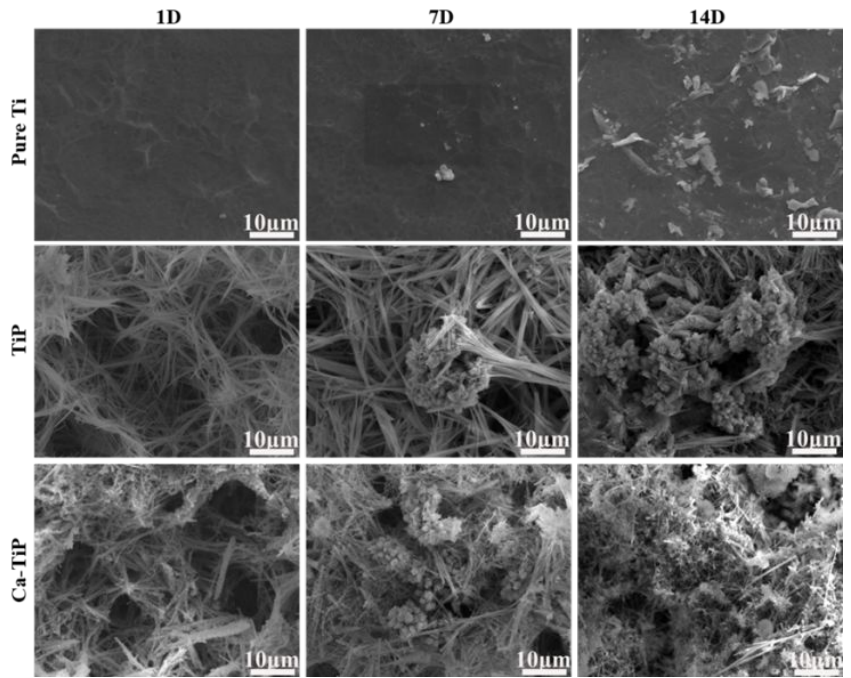

Fig. S4. SEM images showing the apatite formation ability of different Ti surfaces after soaking in SBF for 1 , 7 and $14 \mathrm{~d}$.

\section{Apatite Forming Ability}

It has been suggested that the in vitro apatite-forming ability of $\mathrm{Ti}$ substrates in SBF is consistent with their in vivo bone-bonding behavior and that apatite formation is the decisive factor in osseointegration $[1,2]$. Fig. S4 depicts the morphologies of samples immersed separately in SBF for 1, 7 and 14 days. After 1-day soaking in SBF, only few apatite deposits formed on $\mathrm{Ca}$ TiP coating, but large amount of apatite with a needle-rod structure showed up with the elongation of immersion time. EDS analysis at 14-day immersion (Fig. S5) suggests the $\mathrm{Ca} / \mathrm{P}$ ration of apatite formed on the coating is about 1.60 , much close to that in the mineral phase of hydroxyapatite (1.67). In contrast, nearly no or only small amount of apatite are observed on the pure Ti or TiP coating after immersion in SBF up to 14 days. This is because $\mathrm{Ca}$ ions with an affinity to phosphatidylserine (a phospholipid membrane component) can promote biomineralization by promoting the formation of apatite [3]. Moreover, it seems that the surface microstructure of hydrothermally treated coating provides more nucleation sites for apatite formation. Previous studies have demonstrated that the formation of hydroxyapatite on the implant-bone interface can enhance fibronectin adsorption to improve cell adhesion, spreading, proliferation and differentiation of osteoblasts [4]. Furthermore, it has also been reported that $\mathrm{Ca}$ ions can up-regulate the expression of bone-related genes (e.g., osteocalcin, alkaline phosphatase and type I collagen) to promote bone formation $[5,6]$.

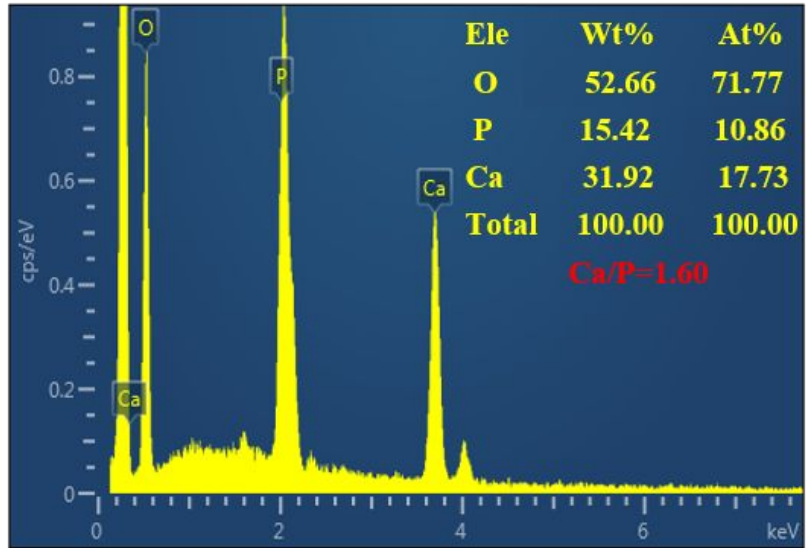

Fig. S5. The EDS elemental analysis of the apatite formed on Ca-TiP after soaking in SBF for 14 days.
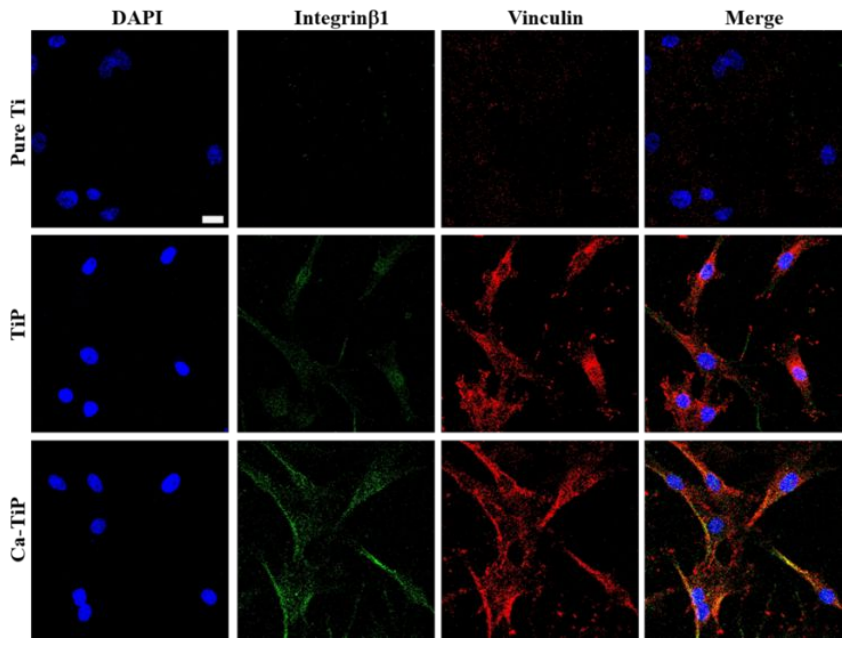

Fig. S6. The expression of Integrin? 1 and vinculin after $48 \mathrm{~h}$ incubation in each group observed by CLSM. 

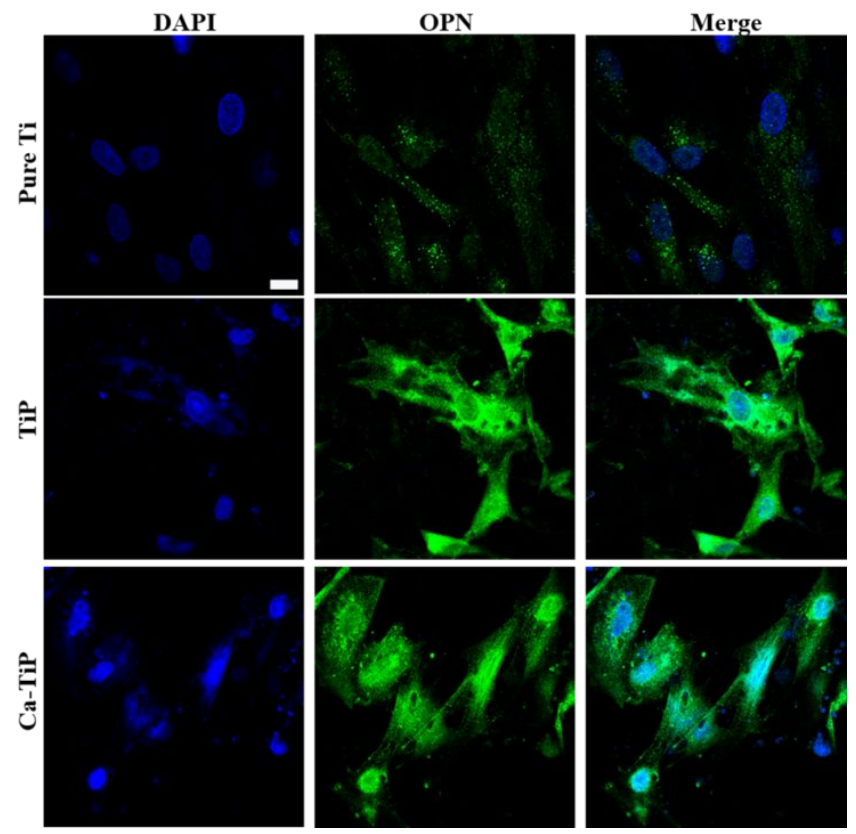

Fig. S7. The expression of OPN after $48 \mathrm{~h}$ incubation in each group observed by CLSM.

\section{REFERENCES}

1. Wang X X, Yan W, Hayakawa S, et al. Apatite deposition on thermally and anodically oxidized titanium surfaces in a simulated body fluid. Biomaterials, 2003, 24: 4631-4637.

2. Xue W, Liu X, Zheng X, Ding C. In vivo evaluation of plasmasprayed wollastonite coating. Biomaterials, 2005, 26: 34553460 .

3. Genge B R, Wu L N Y, Wuthier R E. Mineralization of annexin5-containing lipid-calcium-phosphate complexes-modulation by varying lipid composition and incubation with cartilage collagens. J. Biol. Chem., 2008, 283: 9737-9748.

4. LeGeros $\mathrm{R}$ Z. Calcium phosphate-based osteoinductive materials. Chem. Rev., 2008, 108: 4742-4753.

5. Nayab S N, Jones F H, Olsen I. Effects of calcium ionimplantation of titanium on bone cell function in vitro. J. Biomed. Mater. Res. Part A, 2007, 83: 296-302.

6. Sawada R, Kono K, Isama K, et al. Calcium-incorporated titanium surfaces influence the osteogenic differentiation of human mesenchymal stem cells. J. Biomed. Mater. Res. Part A, 2013, 101: 2573-2585. 